\title{
Silicon Heterojunction and Half-Cell configuration: optimization path for increased module power
}

\author{
F.Gérenton, S.Harrison, J.Eymard, P.Lefillastre, R.Varache, V.Nguyen, D.Munoz, C.Roux \\ CEA-INES, Department of Solar Technologies, F-73375 Le Bourget Du Lac, France
}

\begin{abstract}
Half-cell integration is a smart way to improve module output powers without modifying the global cell architecture. If shingle/half-cell is highly used in standard PV cell technologies, this configuration appears much more challenging when high passivation cell concepts are considered. Indeed, a strong cell degradation is systematically observed for example on silicon heterojunction (SHJ) solar cells after cutting, mainly because of the un-passivated edge created. This paper intends to present recent developments done at CEA-INES on the SHJ halfcell topic. In particular, insights on loss mechanisms and cleavage process conditions are presented, allowing the optimization of the laser cutting process. Best cells were then successfully integrated into a 120 half-cell module, delivering a very high $348 \mathrm{~W}$ output power under standard conditions. Final power gain of $\sim+7 \mathrm{~W}$ was estimated on this module thanks to the specific half-cell architecture demonstrating that half-cell configuration is also fully adapted to the SHJ technology. Finally, hints on further cell performance improvement paths are introduced, with the use for example of localized re-passivation processes to minimize cutting induced degradations.
\end{abstract}

Index Terms - amorphous materials, heterojunction, half-cell, silicon, module.

\section{INTRODUCTION}

The amorphous/crystalline silicon heterojunction (aSi:H/c$\mathrm{Si}$; SHJ) is one of the most attractive solar cell architecture, combining high performance, simple and low temperature processes compatible with high throughput and high module bifaciality [1]-[2]. High performance SHJ-based modules are already among the best PV products available. CEA-INES announced recently for example the realization of a $410 \mathrm{~W}$ module for a 72 SHJ cell Glass-Back-sheet configuration [3]. To improve even further the module performances without modifying the intrinsic cell configuration, one of the most promising paths is the use of Half-Cell or Shingling technologies [4]. However, the manufacturing of such new module concepts can be challenging for high passivation based devices. Indeed, the cutting of the finalized cell leads to the generation of un-passivated trench edges, which degrades significantly the overall SHJ cell performances. Coupled with generally lower overall cell current than for typical hightemperature concepts, this raises questions about the real interest of half-cell configuration for such SHJ devices, as power module expected gains might be counter-balanced by initial cell losses. Thus, we introduce in this paper progress made on industry compatible laser-based cutting processes to minimize the device losses. Specific half-cell characterization method is even applied to discriminate accurately the most promising cutting processes [5]. Complementary experiments are also conducted to highlight clearly the main performance degradation root cause, allowing thus the definition of potential further improvement paths.

Finally, a 120-half-cell module was fabricated with a set of high performance industrial SHJ full cells in order to evaluate the real final power gain we can expect with an optimized integration of such devices in half-cell configuration.

\section{LASER CUTTING OPTIMIZATION}

In this work, SHJ cells were all produced on the CEA INESLABFAB production pilot-line [6], from $156.75 \times 156.75 \mathrm{~cm}^{2}$, pseudo-square wafers, with two possible metallization schemes (4 busbars or busbarless). The cutting process is performed by laser scribing and subsequent mechanical cleavage, using an infrared (1064 nm) Nd:YAG pulsed laser (10 ns pulses). In this section, the optimization of the laserrelated parameters is done by evaluating directly the cut cells electrical performances.

The optimization of the laser-based cutting process is done in two steps. First, the mechanical cleavage feasibility of the cell for different laser grooving conditions is checked, and the incident laser energy is progressively decreased until reaching the minimal required groove depth for an efficient cleavage. Then, current-voltage (IV) characterization is performed before and after the cleavage step for each laser condition. From these measurements, the edge specific recombination current J02edge is extracted [7], as well as the relative losses on main IV parameters. To illustrate the laser optimization impact on cell performances, J02edge and Voc losses for three different laser conditions are presented in Fig.1 for a given set of laser speed and frequency $(10 \mathrm{kHz}, 20 \mathrm{~mm} / \mathrm{s})$. "High power" stands for a non-optimized laser pulse energy (36A). "Reference" is the optimized case, using a single laser pass with minimized laser energy (25A). Finally, "multipass" uses two passes instead of one, allowing further laser pulse energy reduction ( 24A).

The strong drop of J02edge from "high power" to "reference" processes indicates a clear decrease of the recombination on the edge just by minimizing the incident laser energy. Losses in Voc and FF follow the same trend. 
Further laser energy decrease is impossible since the laser groove depth would then be insufficient to allow proper cell cleavage. Alternative approach would then be the introduction of a "multipass" laser condition: two successive laser passes with identical process conditions are applied, allowing for each pass a slight additional reduction of the already optimized incident laser energy, without compromising cell mechanical cleavage properties. A clear gain in both Voc and J02edge is observed again, thanks to the slight power decrease, and despite the quite similar final trench depth. We highlight thus the relevance of the multipass approach, and the major impact of the incident laser energy on final device properties.

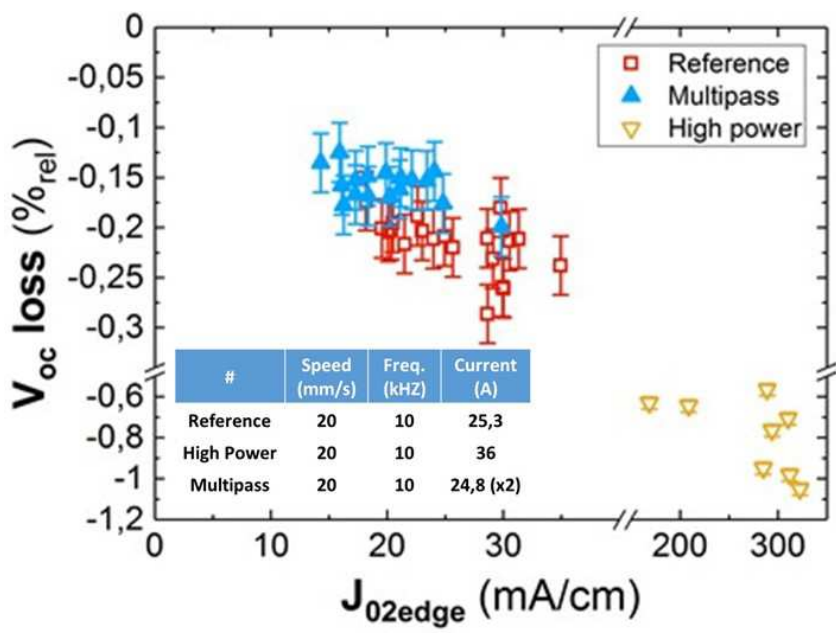

Fig. 1. Half-Cell measurement: Voc losses vs. J02edge for three different cutting processes.

Similar experiment was then conducted for four other laser conditions, with significantly different laser speeds and frequencies, to assess their respective influence on cutting losses (Fig. 2a).

Pulse energy is independently minimized for each condition, as explained in previous section. Very different final optimized laser powers (Current) are obtained for each set of parameters, as both speed and frequency influence greatly material ablation and substrate heating induced by the laser. However, the measured Voc and efficiency losses are quite identical for all the different laser processes tested, demonstrating that with our laser tool, changing the laser speed and working frequency does not influence drastically the edge losses. Laser groove is found to be similar for all laser conditions tested, also confirming that global laser energy applied is very similar for all optimized process conditions. Consequently, laser incident energy remains the main adjustment parameter, and in all cases, a minimum global absolute efficiency loss of $\sim-0.3 \%(\sim-1.3 \%$ relative $)$ is systematically observed on final SHJ half-cells, whatever laser optimization path is considered.

\begin{tabular}{|c|c|c|c|}
\hline \# & $\begin{array}{c}\text { Speed } \\
(\mathrm{mm} / \mathrm{s})\end{array}$ & $\begin{array}{c}\text { Frequency } \\
(\mathrm{kHz})\end{array}$ & $\begin{array}{c}\text { Current } \\
(\mathrm{A})\end{array}$ \\
\hline Laser 1 & 20 & 7 & 25 \\
Laser 2 & 60 & 7 & 28 \\
Laser 3 & 60 & 20 & 29.5 \\
Laser 4 & 80 & 7 & 31 \\
\hline
\end{tabular}

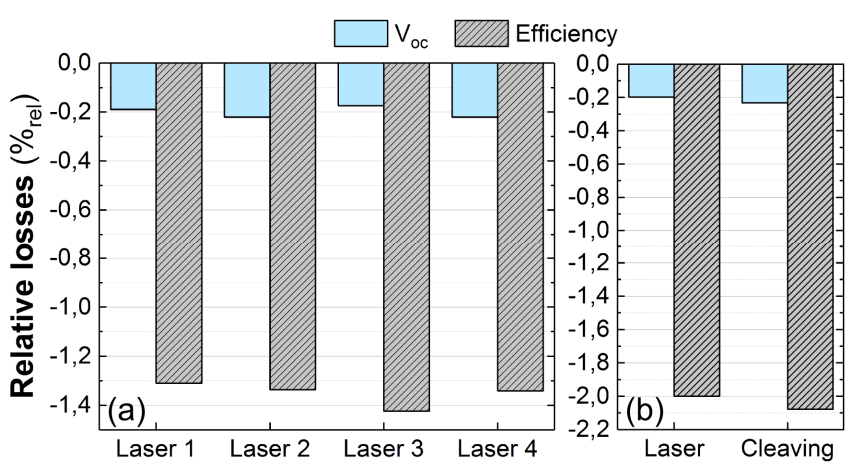

Fig. 2. (Top) Table of alternative laser parameters tested for HalfCell cutting step optimization. (Bottom) Relative losses for (a) Voc and efficiency for 4 different laser processes (half-cell configuration). (b) best laser process and mechanical cleavage only (diagonal cutting configuration).

In order to understand the relative importance of both unpassivated edge and specific laser-induced damage (active layer and silicon volume degradation near the edge) on final cell performance losses, two processes are compared: the "multipass" process as described in Fig. 1, and a manual mechanical cleavage initiated with a diamond tip. As the most fragile crystalline plan on a standard solar wafer is in the diagonal, this specific cleavage axis is chosen, and laser process adapted accordingly. IV losses are evaluated again by characterization of the cells before and after cutting (Fig. 2b). In this case, both sub-cells obtained are reassembled under the IV solar simulator to form a full solar cell, which enables easier IV measurement. Complementary photoluminescence (PL) measurements are realized on the cut cells to highlight eventual edge losses differences (Fig 3.).
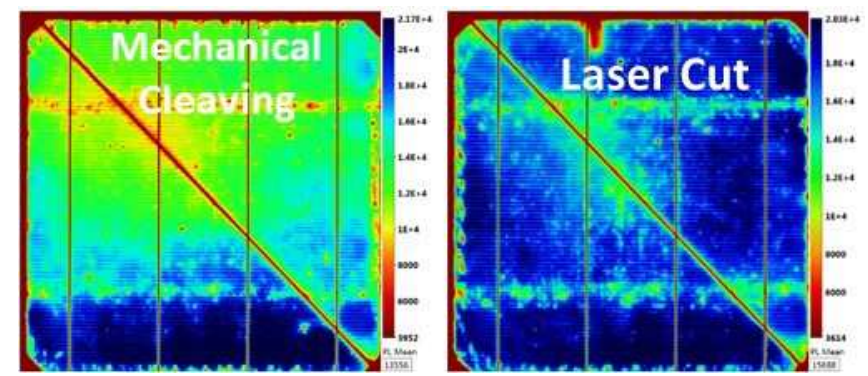

Fig. 3. Examples of PL images of reassembled half-cells: comparison of mechanical wafer cleavage (left), and laser-based cut (right). 
The IV losses are quite similar for laser-cleaved and manually cleaved cells, and even slightly superior for manual cleavage $(-0.20 \%$ with laser and $-0.23 \%$ with manual cleavage in Voc, same trend for efficiency). The PL images do not show any significant differences around the wafer cut edge. This result shows that with a proper laser power optimization as reached in this study, the defects generated by the laser shot and by a mechanical cleavage are almost identical. The defects induced by the laser shot (silicon melting and amorphisation, impurity diffusion...etc) do not translate in an increased edge recombination. If several effect may coexist, the unpassivated wafer edge created is sufficient to generate most of the recombination centers causing the device's performance drop. This also highlights that a standard laser equipment (ns-pulsed, IR) is sufficient to limit the edge losses in the specific case of HET architecture.

\section{SHJ HALF-CELL MODULE INTEGRATION}

A set of high performance BusbarLess SHJ cells is produced on the pilot line for integration into a final 120 Half-Cell module. Cells were all cut by IR Laser with optimized conditions as described in section 2. All sub-cells created were then measured in order to reject the most degraded devices.
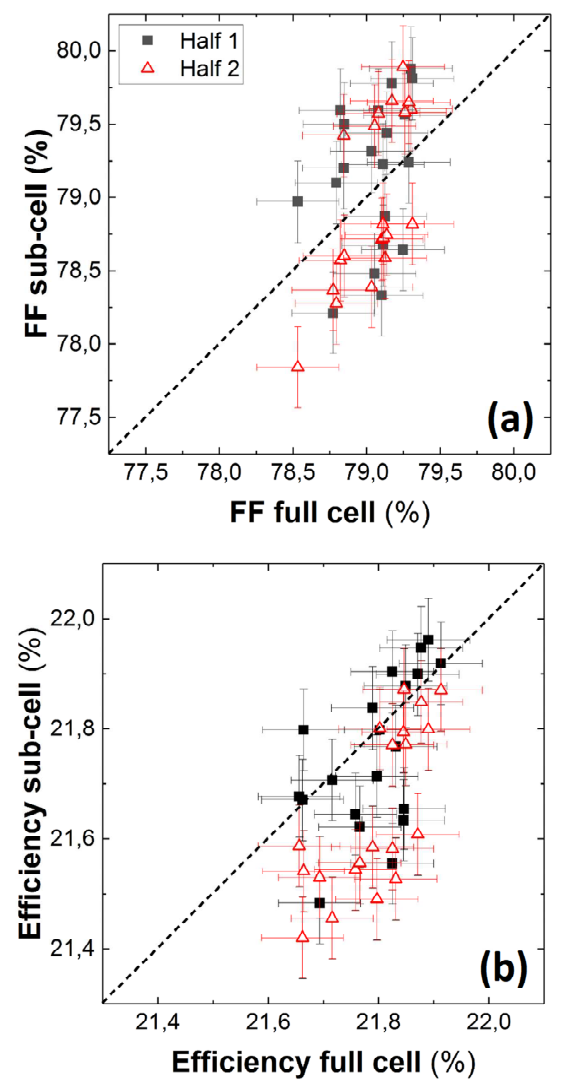

Fig 4. Final sub-cell FF and Efficiency are compared with initial parent cell values. In some specific cases, both sub-cell can show either significantly improved or degraded performances because of localized defectivity and inhomogeneity.

Global post-cleavage measurement shows an increased efficiency distribution dispersion. This is coherent with potential slight laser repeatability issues, and the observation that the final two sub-cells performances can be significantly different from the parent cell (see Fig. 4). If most of the produced half-cells end up with a global uniform efficiency degradation, some sub-cells can surprisingly show improved performances after cutting. This can also be illustrated with the specific FF measurement (Fig. 4) and highlights the fact that the mother cell can sometimes be not perfectly homogenous (local defectivity, layer uniformity, etc.). Indeed, an improved sub-cell is in most cases paired together with a sister-sub-cell showing a stronger degradation than usual.

The global increase in sub-cell dispersion described above emphasizes on the critical need for accurate IV measurements and sorting done directly on the sub-cells after the cleavage step. Best 120 generated sub-cells were then selected out of the global initial available devices (Fig. 5). An average efficiency of $23.3 \%$ is obtained with satisfying current matching. Finally, a SHJ Half-Cell module was fabricated on industrial smart-wire (SWCT) stringer located in the Meyer Burger's facilities in Thun (Switzerland). A very high output power of $348 \mathrm{~W}$ was measured (calibration done with externally certified SHJ module). This result is to our knowledge among the best power ever disclosed for SHJ based modules. When comparing the total half-cell power and the final module output with usual numbers obtained for standard module configuration, $\mathrm{a}+7 \mathrm{~W}$ power gain can be attributed to the specific half-cell configuration (high FF CTM of $98.6 \%$ for example achieved). These different results thus fully confirm the interest of this specific module architecture even for highly passivated architectures such as SHJ.

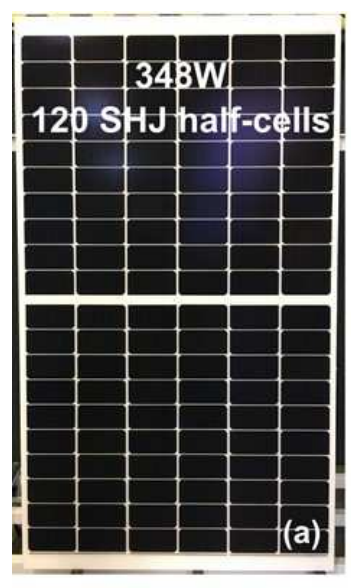

\begin{tabular}{|c|c|c|}
\hline & Pmax (W) & FF\% \\
\hline $\mathbf{1 / 2}$ cells & 340,7 & 81,62 \\
\hline Module & 348,04 & 80,49 \\
\hline CTM & $102,15 \%$ & $98,62 \%$ \\
\hline
\end{tabular}

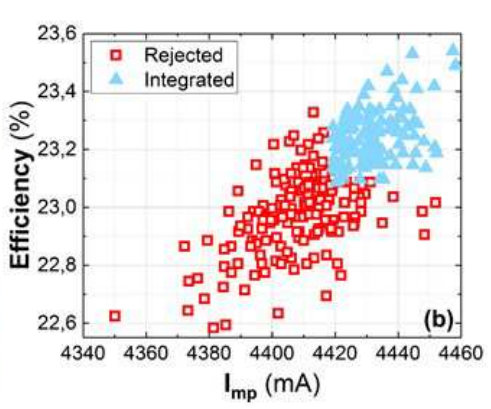

Fig 5. 120 SHJ Half-Cell module. A very high output power of $348 \mathrm{~W}$ is measured. A gain of $\sim+7 \mathrm{~W}$ is obtained thanks to the specific Half-Cell module configuration. 
The CTM power of $102.1 \%$ is the result of a CTM in Isc of $104.1 \%$, combined with a CTM in Voc of $99.6 \%$ and a CTM in FF of $98.5 \%$. Details of losses and gains at module level are summarized in Fig. 6. Changes in short-circuit are explained by global optical stack effects (glass, encapsulant and foil reflection and absorption, foil/cell coupling by index matching, $-6.9 \mathrm{~W})$, by effective shading of grid and interconnection (wire shading and coupling, finger coupling with air/glass interface, $-1.2 \mathrm{~W}$ ), and mainly by white EVA coupling of inter-cell areas $(+21.9 \mathrm{~W})$. Changes in Fill Factor are the results of Joules losses, from finger, interconnection wires and string ribbons $(-6.4 \mathrm{~W})$. Electrical mismatch loss is calculated with the model of Bucciarelli et al [8], and is found to have little influence on power due to fine sorting of cells ($0.5 \%$ ). Finally, change in Voc accounts for $-1.7 \mathrm{~W}$ power loss. These Voc losses are not fully understood yet, and may come from accuracy of initial experimental measurements at cell level or slight increase of edge recombination occurring during module integration.

When considering in details the typical waterfall charts obtained for Full-Cell and Half-cell module configuration, it appears that differences come mainly from white EVA coupling and cell-interconnection joule losses. Firstly, for fullcell architecture, white EVA coupling is lowered to $2 / 3$ of half-cell calculated gain $(+14.6 \mathrm{~W}$ instead of $+21.9 \mathrm{~W})$, because of a greater ratio of perimeter $(\mathrm{x} 4 / 3)$ and a cell area two times bigger. Secondly, Joule losses in wires and ribbons interconnector are similar and four times greater for full-cell (12.4W total loss calculated for full cell configuration) because of the doubled current flowing in the cell. Thus, theoretical power gain when switching from full to half-cell module configuration is $\sim 16 \mathrm{~W}$.

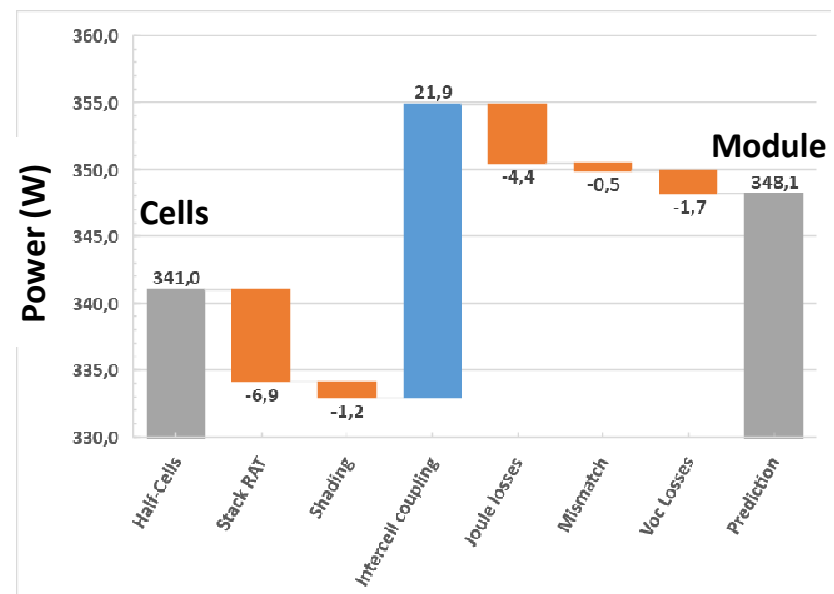

Fig 6. Waterfall chart of power gain/losses for the 120 SHJ HafCell module presented in figure 5. Module power prediction (348.1W) is very close to experimental measurement (348.0W).

\section{CONCLUSION AND PERSPECTIVES}

In this work, we present the latest CEA-INES results obtained for SHJ devices in a half-cell configuration. We focused mainly on IR laser-cutting processes with the following main learning: several different optimization paths are possible for a given laser, as long as a satisfying compromise can be found between minimizing the laser energy/heating while removing enough silicon for an easy cleavage. Indeed, most of the cell's performance degradation arises then from the unpassivated trench edge created, resulting in final incompressible cell efficiency degradation of $\sim-0.3 \%$ abs for the best IR laser processes. A 120 Half-Cell module has been manufactured with a set of high efficiency initial SHJ cells produced on the CEA-INES industrial pilot line. An estimated gain of $+7 \mathrm{~W}$ is obtained thanks to the halfcell configuration, leading to a final module output power of $348 \mathrm{~W}$. To improve further the cell/module performances, repassivation of the opened cell trench appears to be the most promising path. Usual deposition techniques cannot be applied on such finalized devices, because of chamber or temperature incompatibility issues, or parasitic deposition over metallization contact areas. Post metallization spatially localized technique has been tested [9], with an AlOx-like layer deposition. First experiments show indeed encouraging results, with improved cut-cell characteristics on small size samples (Fig. 7).

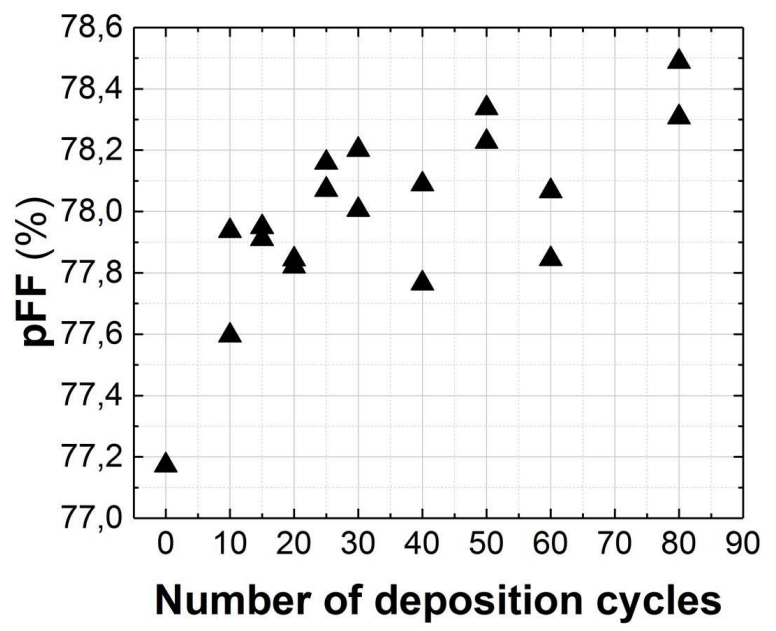

Fig 7. Edge-localized AlOx deposition on $2 \times 2 \mathrm{~cm}^{2}$ laser-cut SHJ samples. Improved pFF is measured with increased layer thickness, proving the efficient edge-repassivation process.

\section{ACKNOWLEDGMENTS}

Authors would like to thank: Meyer-Burger for the Module realization, H2020 Europeans projects DISC and Ampere and French ANR project Despatch for the fundings. 


\section{REFERENCES}

[1] M. Taguchi et al, "24.7\% Record Efficiency HIT Solar Cell on Thin Silicon Wafer", IEEE J. of Photovoltaics, Vol. 4-1 (2014), pp. 96-99.

[2] K. Yoshikawa et al., "Silicon heterojunction solar cell with interdigitated back contacts for a photoconversion efficiency over 26\%", Nature Energy, Vol. 2-5 (2017), pp. 17032.

[3] CEA Press Release: http://liten.cea.fr/cea-tech/liten/en/Pages/ News/ record-photovoltaic- heterojunction.aspx

[4] H. Schulte-Huxel et al., " High-Efficiency Modules With Passivated Emitter and Rear Solar Cells-An Analysis of Electrical and Optical Losses », IEEE J. of Photovoltaics, vol. 7, no 1, p. 25 31, janv. 2017.
[5] F.Gérenton et al, "Edge losses from cutting processes on silicon heterojunction solar cells", to be published.

[6] A.Danel et al, Silicon Heterojunction cells with open circuit voltage over $750 \mathrm{mV}, 35^{\text {th }}$ EUPVSEC, Belgium (2018).

[7] A. Fell et al, "Modeling edge recombination in Silicon solar cells", IEEE J. of Photovoltaics, vol.8, n², pp 428-434.

[8] L.Bucciarelli Jr et al, "Power loss in photovoltaic arrays due to mismatch in cell characteristics", Solar Energy, vol.23, No4, pp.277-288, 1979.

[9] D. Munoz-Rojas et al, "Spatial atmospheric atomic layer deposition: a new laboratory and industrial tool for low-cost photovoltaics", Mater. Horiz 1(3), 314, 2014. 